\title{
Postlaryngectomy Tracheal Cast
}

\author{
Anil Poddar, Piyush Kedia \\ Consultant, Department of Surgical Oncology, BP Poddar Hospital and Medical Research Limited \\ AMRI Hospital, Kolkata, West Bengal, India
}

Correspondence: Anil Poddar, Consultant, Department of Surgical Oncology, 82/3 A, Ballygunge Place, Kolkata-700019 West Bengal, India, Phone: +91 332440 9060, +91 9831004007, Fax: +91 332399 7009, e-mail: dranilpoddar@ hotmail.com

\section{ABSTRACT}

Tracheal cast formation postlaryngectomy is common in patients with dry, inspissated secretions, more so in dry weather, but is rarely large enough to cause significant respiratory distress. Our patient had significantly large tracheal casts to cause respiratory distress even with a widely patent tracheostoma.

Keyword: Tracheal cast.

\section{CASE REPORT}

A 75-year-old male underwent wide field laryngectomy with bilateral neck dissection in M ay 2009 for a T4NOM 0 squamous cell carcinoma of larynx with a TEP. This was followed by adjuvant radiation, about $70 \mathrm{~Gy}$ which concluded in A ugust 2009.

He presented to the emergency one day in the first week of December 2009 with severe respiratory distress. On clinical examination, there was hardly any blast of air through the tracheostoma, though the tracheostoma was widely patent. On inspection, some dry, inspissated mucinous material could be seen deep dow $n$ into the trachea with difficulty. He was immediately taken to the endoscopy suite where his $\mathrm{SpO}_{2}$ on room air was 93 to $94 \%$. A flexible fiberoptic bronchoscope was inserted into the trachea through the tracheostoma, and to our utter surprise, there was a large plug of yellowish green foreign body in the form of a tracheal cast nearly completely occluding the tracheal lumen (Fig. 1). The bronchoscope was removed

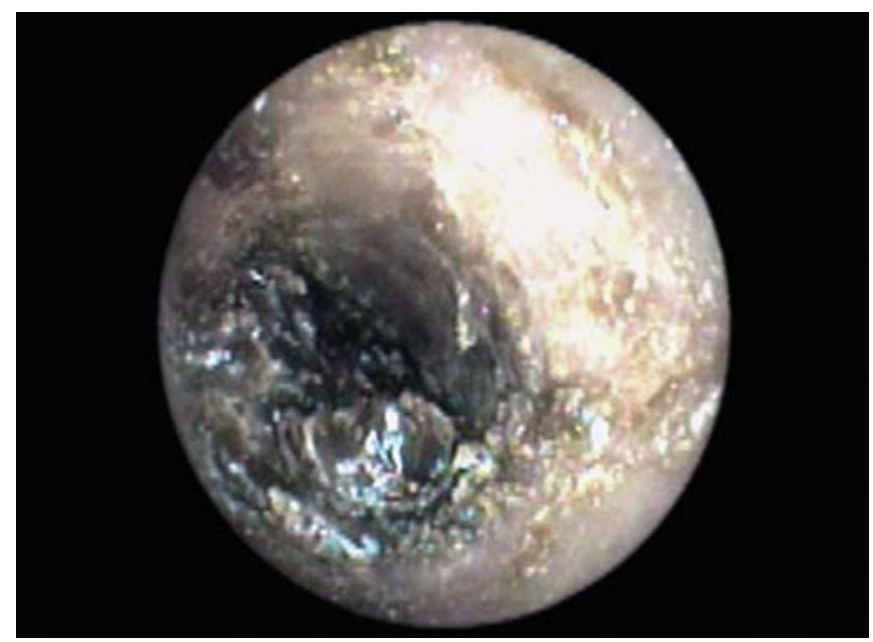

Fig. 1: Tracheal cast inside the trachea-bronchoscopic view and the tracheal cast was removed with a long Magill's forceps. The cast was nearly cylindrical in shape and was almost $3 \mathrm{~cm}$ long (Fig. 2). Patient became immediately comfortable. The $\mathrm{SpO}_{2}$ improved to $100 \%$. Repeat bronchoscopy did not show any block anywhere. The patient was sent home on antibiotics. Histopathological examination showed fibrin with mucus clot. Chest X-ray was normal.

Five days later, the patient presented again in the same condition. A tracheal cast was extracted again from the trachea of a nearly similar size. This time the patient was admitted to the hospital. A long with intravenous antibiotics, he received 2 hourly nebulizations with 1\% Soda bicarb solution. Gradually the cast formation inside the trachea decreased, though the thickness of the tracheal secretions persisted.

He was fitted with a tracheostomy tube and on top of that was fitted with an HM E filter. The function of this filter is to retain the humidity and the temperature of the expired air and allow the inspired air to become humidified. Gradual ly, the patient could go home in about 8 days without further formation of casts. He was also advised to use a room humidifier.

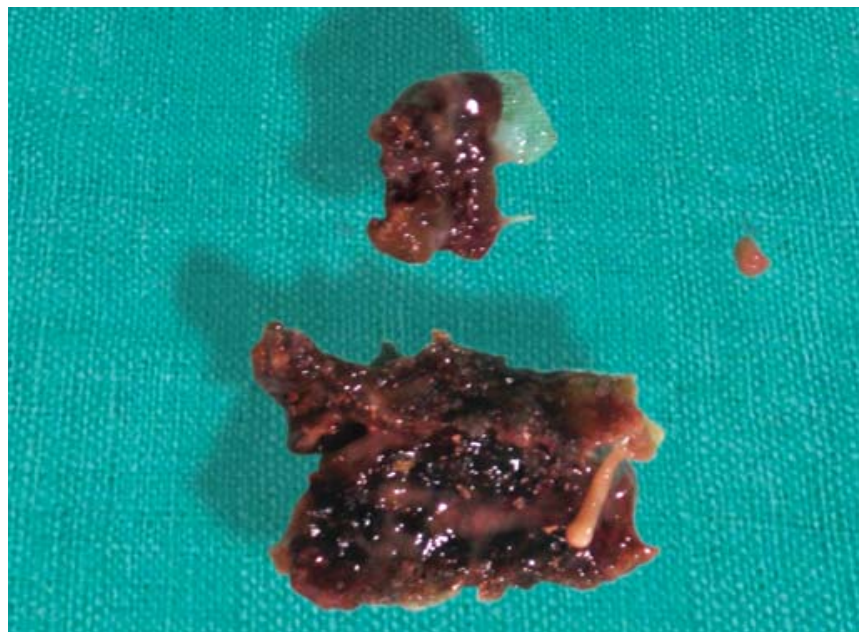

Fig. 2: Casts after extraction 


\section{DISCUSSION}

Tracheal cast formation, postlaryngectomy, especially significantly large ones to the extent of causing respiratory distress are a rare occurrence and have been seldom reported in the literature. A relatively low atmospheric humidity in Kolkata in winter, about 50 to $55 \%$, coupled with an extended radiation portal involving a significant amount of the upper portion of the residual trachea were possibly the chief contributors to the cast formation. A high index of suspicion is imperative when dealing with respiratory distress in a postlaryngectomy patient with a widely patent tracheostoma. Tracheal cast formation is common in dry seasons, though it has al so been reported in the rainy season too. ${ }^{1}$

\section{REFERENCE}

1. Ramanjaneylu P. Postlaryngectomy tracheal cast. Indian J ournal of Otolaryngology 1977;29:45. 